\title{
The ATLAS Strip Detector System for the High-Luminosity LHC
}

\author{
A. Rodriguez Rodriguez, ${ }^{a}$ on behalf of the ATLAS ITk Community \\ ${ }^{a}$ Physikalisches Institut, Albert-Ludwigs-Universität Freiburg, \\ Hermann-Herder-Straße 3, 79104 Freiburg im Breisgau, Germany \\ E-mail: arturo.rodriguez.rodriguez@cern.ch
}

ABSTRACT: The ATLAS experiment at the Large Hadron Collider is currently preparing for a significant upgrade of the Inner Detector (ID) for the High-Luminosity LHC operation, scheduled to start in 2027. A planned integrated luminosity of $4000 \mathrm{fb}^{-1}$ implies integrated hadron fluences over $2 \times 10^{16} \mathrm{n}_{\mathrm{eq}} / \mathrm{cm}^{2}$, requiring a complete replacement of the existing ID. An all-silicon Inner Tracker is under development with a pixel detector surrounded by a strip detector. The strip detector consists of four barrel layers in the centre, while the forward regions are made of six disks at each end, with silicon-strip modules as basic units. With the production of modules scheduled to begin in 2020, a thorough understanding of the current prototype modules is critical. Electrical characterization of the module aims to assess the front-end performance in terms of input noise and noise occupancy. Input noise results from modules built with different front-end designs are presented. Tracking resolutions and detection efficiency are evaluated in beam tests. Several module prototypes have been built and tested in 2018 and 2019. In this report, the beam test results from different module types are presented. Similarly, results from the first double-sided end-cap prototype module, built using a carbon-fibre core with integrated services, are shown. The results focus on hit detection efficiencies and spatial resolution of the modules.

Keywords: Particle tracking detectors (solid-state detectors), Radiation-hard detectors, Performance of High Energy Physics Detectors

${ }^{1}$ Corresponding author. 


\section{Contents}

1 Introduction 1

2 Overview of the ITk Strip Detector 1

2.1 Sensors 2

2.2 Hybrids, Front-End and Powerboard 4

2.3 Local Support $\quad 5$

3 Performance Studies $\quad 5$

3.1 Electrical Tests 5

3.2 Beam Tests 6

4 Summary 9

\section{Introduction}

With the start of the High-Luminosity LHC (HL-LHC) planned for 2027, the ATLAS experiment [1] will see a major upgrade of its Inner Detector (ID). At the HL-LHC, the instantaneous luminosity will be boosted to $7.5 \times 10^{34} \mathrm{~cm}^{-2} \mathrm{~s}^{-1}$ [2], consequently increasing the number of inelastic protonproton interactions per bunch crossing up to 200. This so-called pile-up will push the detector occupancies to unprecedented values demanding an increment of the detector granularity. High radiation doses of up to $2 \times 10^{16} \mathrm{n}_{\mathrm{eq}} / \mathrm{cm}^{2}$ are expected to cause about ten times more radiation damage than for the current ID.

The current ATLAS ID was not designed for such radiation and track density levels. Therefore, a new all-silicon detector, known as the Inner Tracker (ITk), will replace the current ID. The ITk consists of pixel [3] and strip detector systems [2]. Figure 1 shows the schematic layout of the ITk detector. Both systems consist of barrel layers located in the central region around the interaction point and two end-caps located in the forward and backward regions. The design features high modularity and an extension of the pseudorapidity coverage from $|\eta| \leq 2.5$ to $|\eta| \leq 4$. It increases the granularity and intends to reduce the material. The ITk detector, designed with more radiationhard sensors and readout electronics, is expected to at least maintain, if not improve, the tracking performance of the current ID under more demanding conditions.

\section{Overview of the ITk Strip Detector}

The highly modular design allows for multi-site construction, making the building effort worldwide with more than 20 institutes participating in the project. The ITk strip detector consists of four barrel layers and six disks in each end-cap, which follow the same design philosophy. The two inner barrel layers are populated with Short Strip (SS) detector modules while the two outer layers 


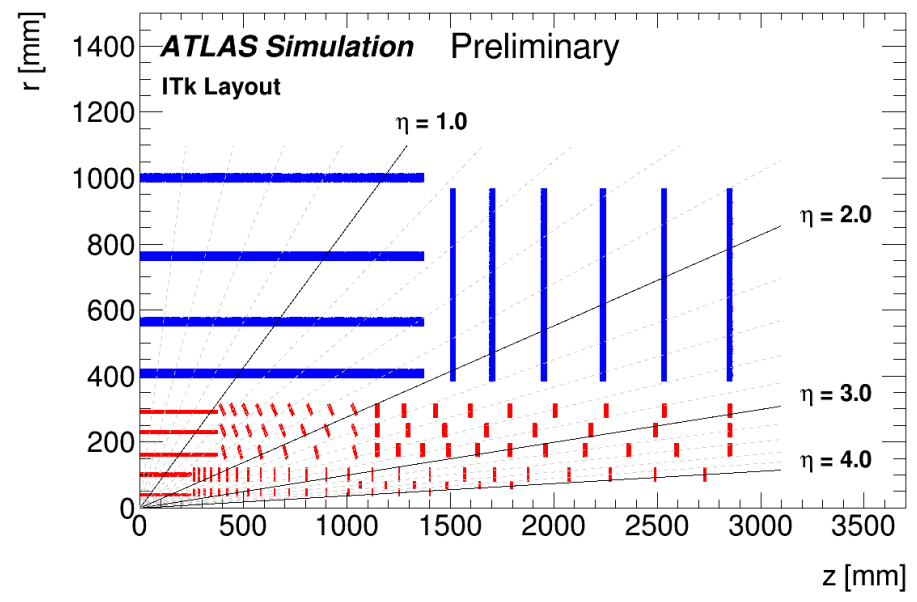

Figure 1: Schematic layout of the ATLAS ITk detector [2]. The active elements of the strip detector are shown in blue, and those of the pixel detector are shown in red. The horizontal axis is along the beam line with zero being the interaction point. The vertical axis is the radius measured from the interaction region. Copyright 2020 CERN for the benefit of the ATLAS Collaboration. CC-BY-4.0 license.

are equipped with Long Strip (LS) detector modules. Each end-cap disk is made of 32 petals, and each petal is equipped with detector modules arranged in six rings.

The smallest building unit is the ITk strip detector module. Barrel and end-cap modules follow the same conceptual design and therefore use comparable components, although there are eight different layouts. The modules are glued onto the support structures with integrated services and cooling. An ITk strip module is built from a silicon sensor and flexible printed circuit boards called hybrids, as well as powerboards which are glued directly onto the sensor. The number of hybrids and powerboards depends on the module type. In the barrel, modules have one powerboard and one or two hybrids. In the end-cap, the modules feature one or two powerboards, and the number of hybrids varies from one to four. Hybrids hold the front-end Application-Specific Integrated Circuits (ASICs) and aggregator chips. Powerboards contain a DC-DC converter, a monitoring and control chip, and a high voltage bias filter.

Figure 2a shows an exploded view of a Short Strip barrel module where the different components are depicted and labelled. Modules are glued back-to-back on the local support structure as shown in Figure $2 \mathrm{~b}$ for the case of a barrel stave equipped with Short Strip modules. In the barrel, modules on each side are rotated by $26 \mathrm{mrad}$ giving a total stereo angle of $56 \mathrm{mrad}$ to allow for two-dimensional space point reconstruction. In the end-caps, the total stereo angle is $40 \mathrm{mrad}$, achieved by a $20 \mathrm{mrad}$ rotation of the strips, which has been implemented in the sensor design [4].

\subsection{Sensors}

The ITk strip detector uses $320 \mu \mathrm{m}$ thick $\mathrm{n}^{+}$-in-p float zone silicon sensors implemented on 6-inch wafers [5]. This type of sensors collects electrons and therefore does not suffer from radiationinduced type inversion observed in n-bulk sensors [7]. In the barrel, sensors have a rectangular shape of about $10 \times 10 \mathrm{~cm}^{2}$ with parallel strips with a pitch of $75.5 \mu \mathrm{m}$. There are two sensor designs, 


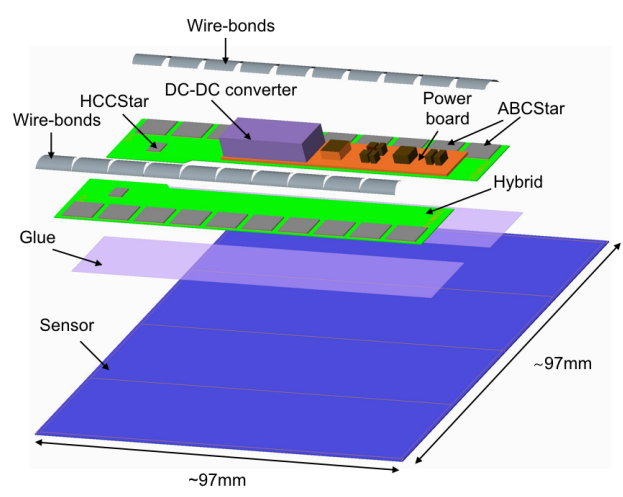

(a)

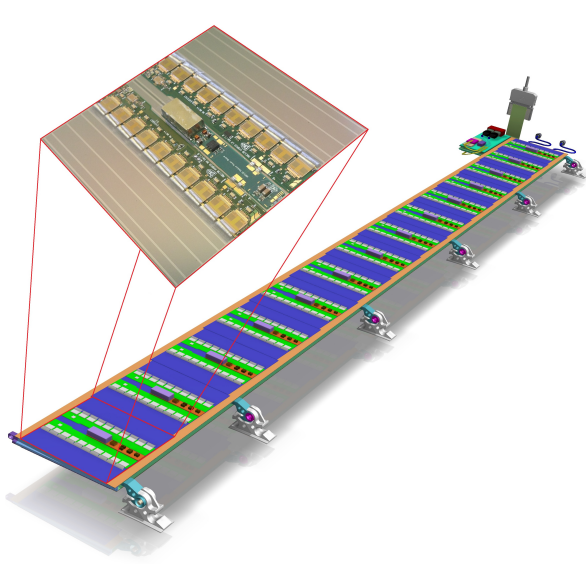

(b)

Figure 2: (a) Exploded view of a Short Strip barrel module with all relevant components. Long Strip and end-cap modules feature the same component groups [2], (b) 3D sketch of a barrel stave equipped with 14 Short Strip modules [6]. A real module is shown with line guides to its position on the stave. Copyright 2020 CERN for the benefit of the ATLAS Collaboration. CC-BY-4.0 license.
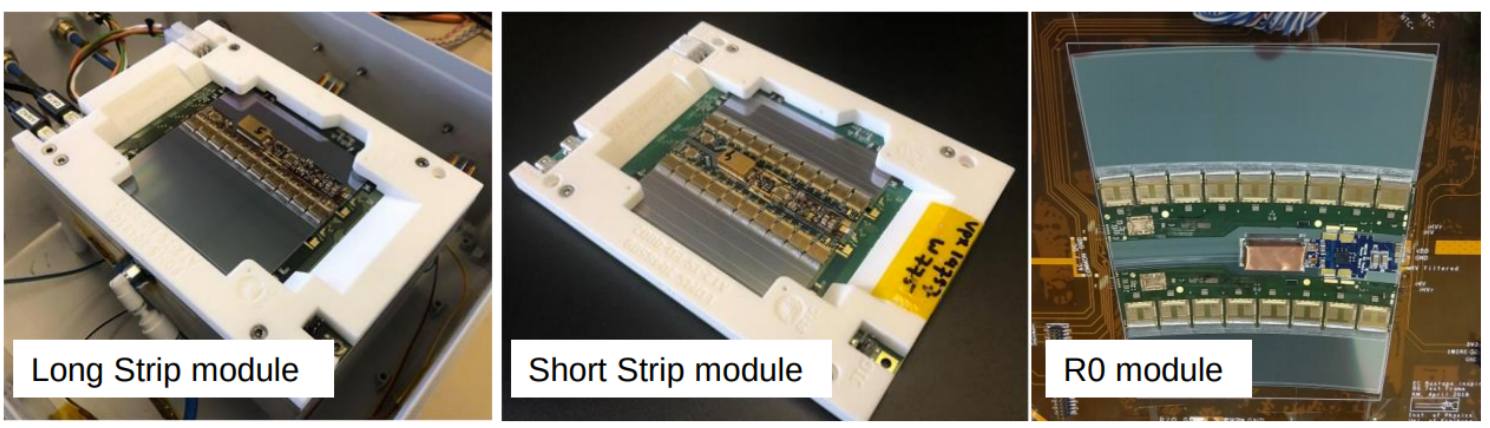

Figure 3: Long Strip and Short Strip modules for the barrel and R0 module for the end-cap. Copyright 2020 CERN for the benefit of the ATLAS Collaboration. CC-BY-4.0 license.

the SS with four rows of $2.4 \mathrm{~cm}$ strips and the LS with two rows of $4.8 \mathrm{~cm}$ strips. In the end-caps, six different designs are used in order to provide R- $\phi$ coverage. Sensors have a stereo-annulus geometry with strips pointing towards a common origin. The strip length varies from 1.5 to 6.0 $\mathrm{cm}$, depending on the expected track density and the pitch size rises from 70 to $81 \mu \mathrm{m}$. Modules are named from R0 (innermost ring) to R5 (outermost ring). Figure 3 shows, from left to right, pictures from a LS, a SS and an R0 module in their test frames. Hybrids (green), ASICs (gold), the powerboard (blue) and the silicon sensor can be easily identified in the picture of the R0 module.

The expected dose for the ITk strip detector is $1.6 \times 10^{15} \mathrm{n}_{\mathrm{eq}} / \mathrm{cm}^{2}$ [2] for an ultimate integrated luminosity of $4000 \mathrm{fb}^{-1}$, which makes testing the performance of the sensor at their end-of-life an essential task. The performance has been tested through extensive irradiation campaigns in the last years. Figure 4 shows the collected charge as a function of the fluence for different forms of irradiation. The measurements were performed using beta sources at a bias voltage of $500 \mathrm{~V}$ for the ATLAS12 (A12) [8] and ATLAS17 (A17) sensor prototypes. It is observed that at the maximum 
expected fluence, the collected charge is still larger than 10000 electrons. The sensors fulfil the requirement of a minimum signal-to-noise ratio of ten at the end-of-life of the ITk strip detector if the noise prediction from [2] is considered.

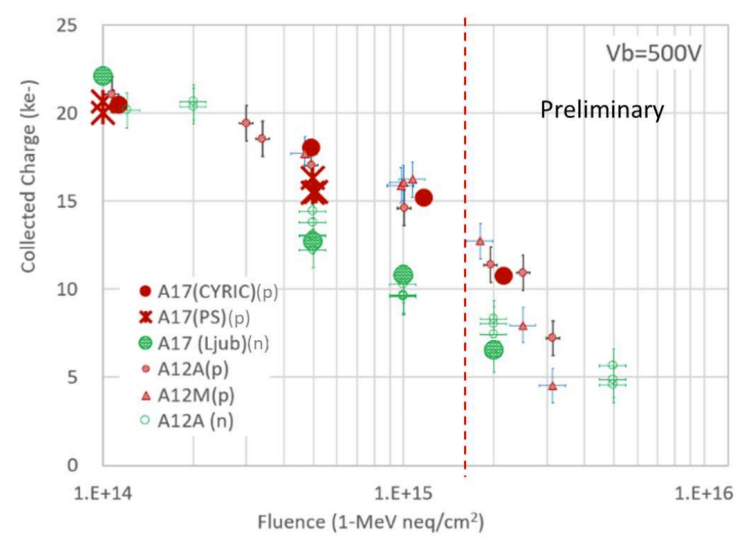

Figure 4: Collected charge of irradiated prototype sensors (A12 and A17) as a function of the irradiation fluence measured at $500 \mathrm{~V}$ [9]. Different irradiation types are colour-coded. The maximum fluence expected at the end-of-life is indicated by the dashed red line. Copyright 2020 CERN for the benefit of the ATLAS Collaboration. CC-BY-4.0 license.

\subsection{Hybrids, Front-End and Powerboard}

Sensors are read out by radiation-hard circuits called hybrids [10, 11]. Hybrids consist of four-layer Kapton PCBs resulting in a flexible circuitry with low mass which carries specially designed and also radiation-hard ASICs, the ATLAS Binary Chip (ABC) [12] and the Hybrid Controller Chip (HCC). Due to sensor geometries, there are a total of 13 different layouts for the end-cap and one unique design for the barrel.

The ATLAS Binary Chip, called ABCStarV0 in its almost final version, is an analogue frontend chip produced in a $130 \mathrm{~nm}$ CMOS process. It provides analogue amplification, shaping, and binary discrimination with individual trimming features to read out 256 strips. The chip architecture permits multi-level-trigger data flow control by storing data from previous beam crossings in an event buffer which can be retrieved later with the reception of the trigger signal. Additionally, the ABCStarV0 has direct communication to the HCCStar changing the previous serial transfer of data (ABC130) and removing bandwidth issues while simplifying the architecture [2].

The HCCStar, also built in a $130 \mathrm{~nm}$ CMOS process, provides all input/output control for itself and the ABCStarV0. It processes trigger types, configuration and calibration commands which are received and passed to the ABCs when required. The HCC aggregates the ABC's data, grouping them in an event and sending them out to the End-of-Substructure (EoS) card, located in each local support structure.

The powerboard provides DC-DC conversion [13], from 11 to $1.5 \mathrm{~V}$, for the front-end. It carries an Autonomous Monitor and Control (AMAC) chip, which provides measurements of temperature, voltages and currents, as well as control of low voltage, power states and a high voltage switch. 


\subsection{Local Support}

Two different support structures provide mechanical stability and services like cooling, power and data transmission. The structures are built from a carbon fibre honeycomb core with a copper/kapton co-cured bus-tape. The bus-tape provides the electrical transmission for power, trigger, timing and control (TTC), data and detector control signals. Cooling is implemented using $\mathrm{CO}_{2}$ and embedded titanium tubes driving the operation temperature to $-35^{\circ} \mathrm{C}$.

The interface from staves and petals to the off-detector electronics is carried out through the EoS card. It sends TTC information to the HCCStars. In addition, bias voltage and detector control signals are sent from the EoS to the AMACs in the modules. Data are sent to the off-detector electronics via optical transceivers with a speed of up to $8.96 \mathrm{Gbit} / \mathrm{s}$ [14]. Modules are glued directly onto the bus-tape, and the electrical connections are made via wire-bonds. Figure 5 shows a barrel stave and end-cap petal prototype. The petal in Figure 5a is fully loaded with modules built using plain silicon sensor dummies, apart from the R0 module, which uses a production-grade sensor. Fully functional hybrids, ABCStarV0 and powerboards are used. The stave in Figure $5 \mathrm{~b}$ is not completely loaded, but all the SS modules used are fully functional.

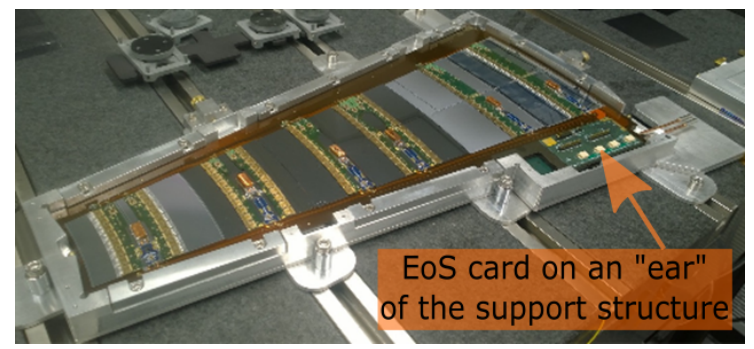

(a)

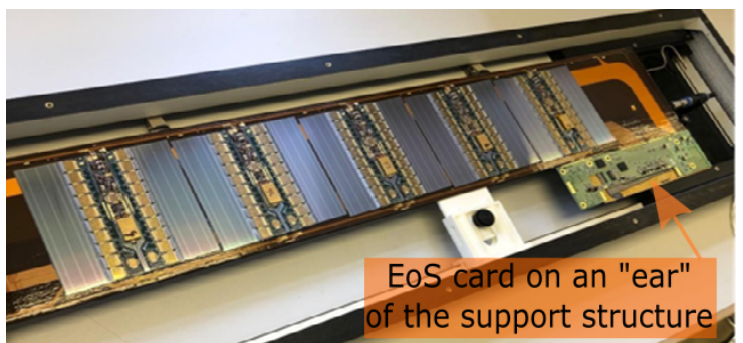

(b)

Figure 5: ATLAS ITk strip local support structures: (a) a fully populated end-cap petal, (b) a partially populated barrel stave. On both pictures the End of Substructure (EoS) card is indicated. Copyright 2020 CERN for the benefit of the ATLAS Collaboration. CC-BY-4.0 license.

With more than 20000 modules to build, the pre-production phase is starting in 2020. Testing the performance of modules with the majority of components in their final design constitutes a milestone. A summary of recent electrical and beam tests results is given in the next section.

\section{Performance Studies}

\subsection{Electrical Tests}

The first stage of module testing corresponds to the electrical tests which are carried out in a cleanroom. The analysis includes, for example, test of powering, correct front-end communication and configuration, and threshold scans to evaluate the linearity of the amplifiers [15]. There are two main analogue tests: response curve and noise occupancy. These evaluate the performance of the module in terms of input noise and noise occupancy.

Figure 6 shows a comparison of the input noise in Equivalent Noise Charge (ENC) per channel for different segments of two R0 modules with different front-end prototypes. The R0 modules 
have four strip segments, which are read out by two hybrids labelled as R0H0 and R0H1. R0H0 has eight readout chips while $\mathrm{R} 0 \mathrm{H} 1$ has nine.
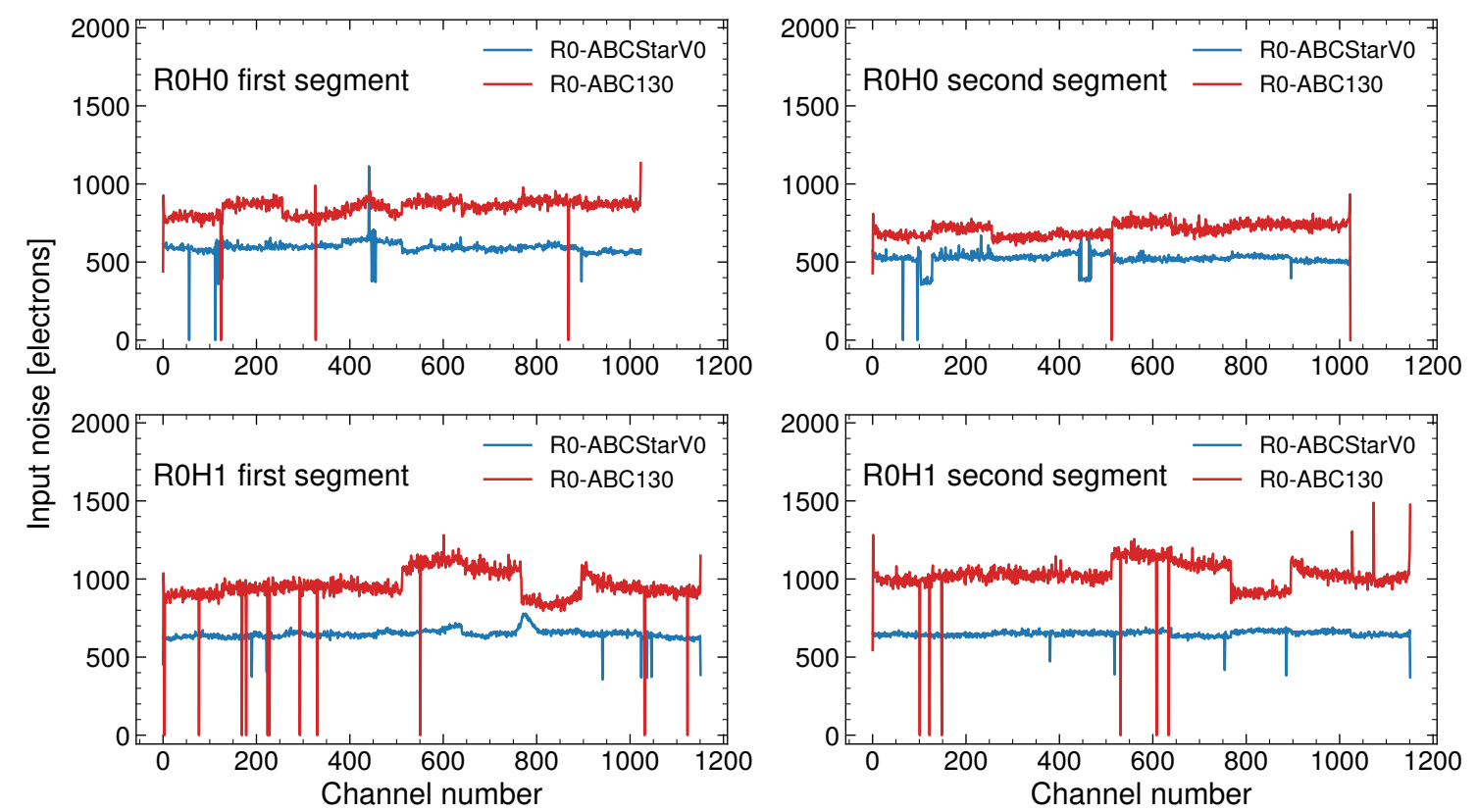

Figure 6: Comparison of the Equivalent Noise Charge (ENC) as a function of the channel number for two R0 modules, equipped with the ABCStarV0 in blue and ABC130 in red. The upper plots show the comparison between the R0H0 hybrid of each module. The lower plots display the comparison for the $\mathrm{R} 0 \mathrm{H} 1$ hybrids. Input noise values of zero electrons corresponds to unbonded channels. Copyright 2020 CERN for the benefit of the ATLAS Collaboration. CC-BY-4.0 license.

It can be observed that the noise distributions show flat shapes, which is an indication of the stability among the channels. The channel-by-channel differences are due to statistical fluctuations of the response curves from where the noise is calculated. The noise values for the ABCStarV0 are well below the 1000 electrons requirement from [2]. For the ABC130, the average input noise is below 1000 electrons, but the values are larger than those for ABCStarV0. The differences in the noise between the two front-ends are linked to common noise induced by the assembly method used for the module with the $\mathrm{ABC} 130$ chipset. The lower input noise values of the $\mathrm{R} 0 \mathrm{H} 0$ with respect to the $\mathrm{R} 0 \mathrm{H} 1$ are related to the smaller coupling capacitance, due to shorter strip lengths for the first and second segments of the R0 module.

\subsection{Beam Tests}

Tracking resolution and hit efficiency have been studied using high energy beam test facilities. During the current LHC long shut down, beam test campaigns have exclusively taken place at the DESY beam test facility [16]. Tests were performed using an electron beam with an energy between 4.0 and $5.8 \mathrm{GeV}$. Tracking reconstruction is achieved with EUDET-type beam telescopes [17] consisting of six silicon pixel planes. In order to place a timing tag on the track, an additional pixel silicon plane, with the same integration time as the ITk modules, is used. The devices under 
test (DUTs) are positioned in the centre of the telescope and are controlled and read out using EUDAQ2 [18].

In 2018 and 2019, several beam test campaigns took place. The results presented in this document correspond to four different modules: the first double-sided R0 end-cap module [15], a non-irradiated LS module and two irradiated LS and R0 modules. With the exception of the double-sided $\mathrm{R} 0$ module, which was built using the $\mathrm{ABC} 130$ prototype, the remaining modules are equipped with the final design Star chipsets.

\section{Data Reconstruction and Analysis}

Track reconstruction is achieved using the EUTelescope software [19]. Tracks are reconstructed only using hits from the silicon pixel planes. A track-based alignment procedure, which also examines multiple Coulomb scattering, is used for each DUT and telescope configuration. The telescope resolution depends on the distance between its detector planes and the energy of the electrons used during the tests [17]. The telescope and beam configuration provides tracking resolutions between 5 and $10 \mu \mathrm{m}$. In the case of end-cap modules, a customized EUTelescope is used to account for the stereo annulus geometry of the sensors, where hit reconstruction and alignment use a cylindrical geometry with its longitudinal axis parallel to the beam axis. As the beam test facility runs in a parasitic mode of the DESY II [16] accelerator, particles do not arrive with a fixed timing with respect to the $40 \mathrm{MHz}$ LHC clock, and a selection of tracks based on time must be performed. In the analysis, a good track must fulfil two criteria. It has to match a hit on the timing plane and be within a few ns of the optimal time when it passed through the DUT. Because of the binary readout, the detector efficiency is defined as the fraction of good tracks that also have a DUT hit. For the ITk, the benchmark is to operate modules at thresholds providing a hit reconstruction efficiency higher than $99 \%$ and noise occupancies below $0.1 \%$. Likewise, a signal-to-noise ratio of ten or higher at the end-of-life satisfies the ITk requirements.

\section{Beam Test Results}

Detector efficiencies are used to set the reference point for the module performance and constitute the central subject of study. Efficiency curves are studied as a function of the threshold set in the front-end in units of digital-analogue-converter (DAC). DAC units are converted to fC using a front-end calibration obtained from direct charge injection in the ASIC channels. For this study, two regions were defined in the sensor based on where the track intercepted the strip. The first region is $15 \mu \mathrm{m}$ wide taking as the centre the middle of two adjacent strips. If the particle passes within it, it is counted as traversing in the so-called inter-strip region or edge. The other particles are counted as hitting the centre of the strip. Figure 7a shows the measured efficiency as a function of the threshold in $\mathrm{fC}$ for the full strip, the centre and the edge regions. The full strip region refers to the combination of the edge and centre regions.

The median charge is defined as the charge corresponding to the threshold where the detector registers half of the tracks. It allows evaluating the overall efficiency. For the full strip area, the median charge is $3.65 \mathrm{fC}$. For the centre, the measured median charge is $3.72 \mathrm{fC}$, and for the edge region, it is $3.37 \mathrm{fC}$. In the ITk, particles will pass through the detector at various angles. Therefore, changing the beam incidence angle is crucial to study the performance of the module. A corresponding efficiency curve is shown in Figure $7 \mathrm{~b}$, where the DUT is mounted with a $12^{\circ}$ angle 
between the normal of the detector plane and the beam axis. As observed in the figure, the result is a median charge of $3.0 \mathrm{fC}$ for all the defined regions. Because of the angle, the charge is now deposited with a higher probability in more than one strip. Due to the nature of the binary readout, the overall efficiency drops because a single strip does not record enough charge to produce a hit above the threshold [20]. This is the well-known charge-sharing effect.

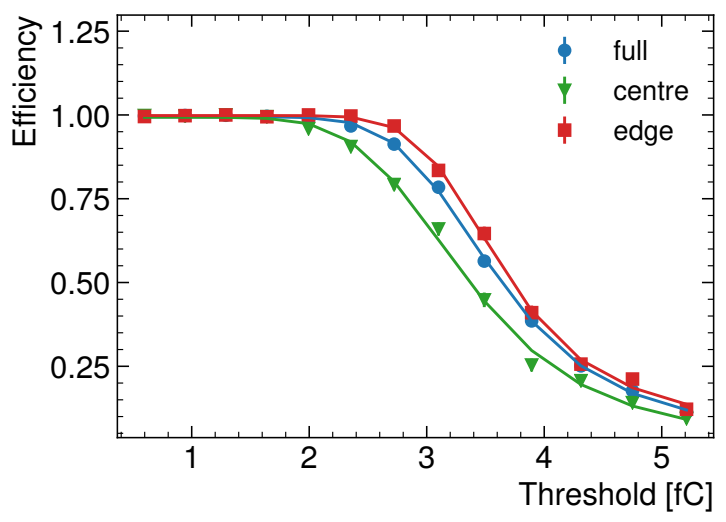

(a)

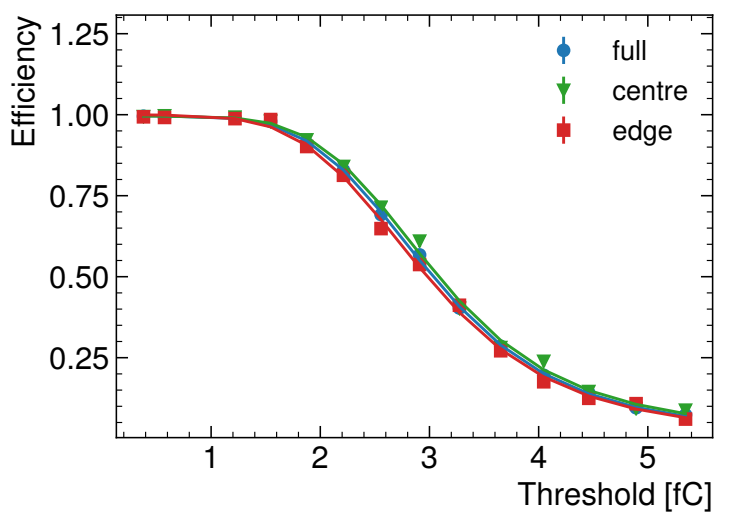

(b)

Figure 7: Detection efficiency of the unirradiated LS module for (a) perpendicular beam incidence and (b) $12^{\circ}$ incidence angle for three regions determined within the sensor. The edge region is $15 \mu \mathrm{m}$ wide taking as the centre the middle of two adjacent strips. The centre is the area between two edge regions and the full area refers to the total strip pitch. Solid curves show a fit to the measured data points using an adapted error function. Copyright 2020 CERN for the benefit of the ATLAS Collaboration. CC-BY-4.0 license.

The double-sided R0 structure consists of two R0 modules glued back-to-back on a small scale petal prototype. The core is built from a carbon-fibre honeycomb with embedded titanium pipes for cooling and a bus-tape on each side. Two fully populated R0 modules are positioned by hand on each side of the core. The latter leads to a stereo angle of $31 \mathrm{mrad}$ between the strips of the two sides instead of the $40 \mathrm{mrad}$ design value. Space points are reconstructed by finding the crossing point between two straight lines on the two modules. For each event, the residual is calculated as the distance between the space point and the extrapolated track position on the modules. The distribution of the radial residual $R$, i.e. the direction along the strips, is shown in Figure 8a.

The overlay of the two strips produces a rhombus-shaped region whose projection is the triangular function seen in the radial residual distribution. The expected radial resolution is given by [20]:

$$
\sigma_{R}=\frac{\sigma_{\phi}}{\sqrt{2} \cdot \sin \left(\phi_{s} / 2\right)} \approx 1.1 \mathrm{~mm}
$$

where $\phi_{s}$ is the stereo angle, and $\sigma_{\phi}$ is the angular resolution of a single module given by $\sigma_{\phi}=$ $p_{\phi} / \sqrt{12}$ with $p_{\phi}$ the strip angular pitch. Figure $8 \mathrm{~b}$ shows the angular residual distribution for the two independent R0 modules. The root-mean-squared (RMS) calculated from the $R$ residual distribution is shown in Figure 8a. This value agrees with the expectation obtained from eq. (3.1). 


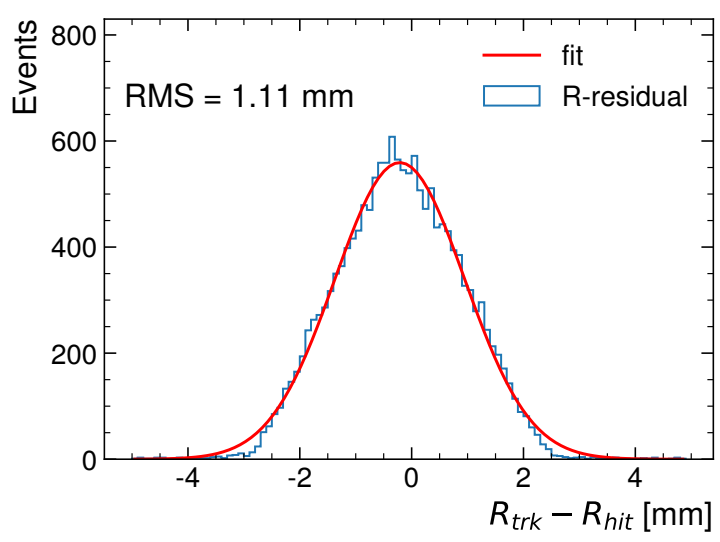

(a)

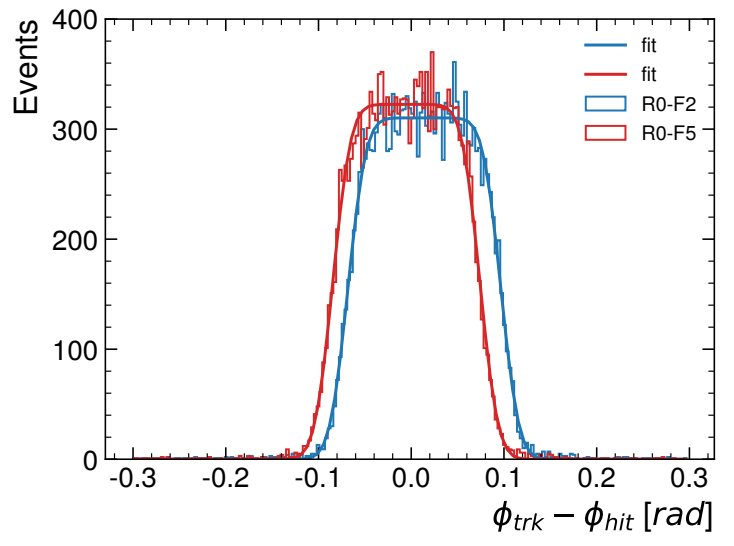

(b)

Figure 8: Residual distributions obtained for the double-sided R0 module. (a) Radial distance distribution along the strips obtained from the space points and the reconstructed tracks from the telescope. (b) Angular distribution for the two individual R0 modules. Copyright 2020 CERN for the benefit of the ATLAS Collaboration. CC-BY-4.0 license.

Figure $8 \mathrm{~b}$ shows the angular residual distribution of the two individual R0 modules, labelled as $\mathrm{R} 0-\mathrm{F} 2$ and R0-F5. RMS values of $\mathrm{RMS}_{(\mathrm{R} 0-\mathrm{F} 2)}=56.5 \mu \mathrm{rad}$ and $\mathrm{RMS}_{(\mathrm{R} 0-\mathrm{F} 5)}=53.1 \mu \mathrm{rad}$ were obtained, in agreement with the $50 \mu \mathrm{rad}$ expectation given by $\sigma_{\phi}=p_{\phi} / \sqrt{12}$.

As mentioned in section 2.1, the ITk strip detector modules will be exposed to very high radiation doses and testing the performance of the irradiated modules at the end-of-life fluence is crucial. Therefore, several irradiation campaigns have taken place to evaluate the performance of different barrel and end-cap modules at the end-of-life dose. Two irradiated modules, a LS and an R0, equipped with the ABCStarV0 front-end have been tested. The LS module was built from an ATLAS17LS sensor that was neutron-irradiated to $5.1 \times 10^{14} \mathrm{n}_{\mathrm{eq}} / \mathrm{cm}^{2}$ and gamma-irradiated Star components (hybrids, front-end, powerboard) to $25 \mathrm{Mrad}$. The R0 module was built from an ATLAS12EC sensor that was proton-irradiated to a fluence of $1.5 \times 10^{15} \mathrm{n}_{\mathrm{eq}} / \mathrm{cm}^{2}$. Star Hybrids, ASICs and powerboard were gamma-irradiated to a dose of $35 \mathrm{Mrad}$. The neutron equivalent fluences equal the maximum expected doses for each type of module, including a safety factor of 1.4. Both modules were operated at a $500 \mathrm{~V}$ bias voltage. The signal-to-noise ratio was measured using threshold scans from 0.3 to $1.0 \mathrm{fC}$. The measured value for the LS module was 15.9 and it was 11.7 for the R0 module. These values are in agreement with the ITk requirement for the end-of-life performance. These are preliminary values, and more detailed studies are ongoing. Nevertheless, both irradiated modules, built with StarV0 components, show a signal-to-noise ratio above the specification.

\section{Summary}

After an extensive program of research and development, and with almost all design decisions made, the ITk strip community is ready to start the pre-production phase during which about $5 \%$ of all modules will be built. Electrical tests prove that the newest front-end design provides reliable 
operation stability and lower input noise values. Beam test studies of prototype modules have demonstrated that the detector design can provide the expected performance. The latter is true for non-irradiated and irradiated modules. Measured signal-to-noise ratios above ten for irradiated modules at a bias voltage of $500 \mathrm{~V}$ prove that the requirements can be satisfied over the full life time of the detector.

\section{Acknowledgments}

The measurements leading to these results have been performed at the beam test facility at DESY Hamburg (Germany), a member of the Helmholtz Association (HGF). We gratefully thank the operators of this facility. This project has received funding from the European Union's Horizon 2020 Research and Innovation program under Grant Agreement no. 654168.

\section{References}

[1] ATLAS Collaboration, The ATLAS experiment at the CERN large hadron collider, JINST 3 (2008) S08003.

[2] ATLAS Collaboration, Technical Design Report for the ATLAS Inner Tracker Strip Detector, CERN-LHCC-2017-005, ATLAS-TDR-025, https://cds.cern.ch/record/2257755.

[3] ATLAS Collaboration, Technical Design Report for the ATLAS Inner Tracker Pixel Detector, CERN-LHCC-2017-021, ATLAS-TDR-030, https://cds.cern.ch/record/2285585.

[4] C. Lacasta et al., Design of the first full size ATLAS ITk strip sensor for the endcap region, Nucl. Instrum. Meth. A 924 (2019) 137.

[5] Y. Unno et al., Development of n+-in-p large-area silicon microstrip sensors for very high radiation environments - ATLAS12 design and initial results, Nucl. Instrum. Meth. A 765 (2014) 80.

[6] Science and Technology Facilities Council Particle Physics Department, ATLAS Inner Tracker Upgrade (ITk), https://www.ppd.stfc.ac.uk/Pages/ATLAS-SLHC-Upgrade.aspx. Accessed: 2020-04-09.

[7] D. Pitzl et al., Type inversion in silicon detectors, Nucl. Instrum. Meth. A 311 (1992) 98.

[8] L. B.A. Hommels et al., Detailed studies of full-size ATLAS12 sensors, Nucl. Instrum. Meth. A 831 (2016) 167.

[9] S. Wonsak, The ATLAS ITk Strip Detector System for the Phase-II LHC Upgrade, The 28th International Workshop on Vertex Detectors, PoS (Vertex2019) 017.

[10] K. Mahboubi et al., The front-end hybrid for the ATLAS HL-LHC silicon strip tracker, JINST 9 (2002) C02027.

[11] C. García Argos et al., Front-end Electronics of the Forward Strip Detector for the ATLAS HL-LHC Upgrade, Topical Workshop on Electronics for Particle Physics, PoS (TWEPP2018) 014.

[12] W. Lu et al., Development of the ABCStar front-end chip for the ATLAS silicon strip upgrade, JINST 12 (2017) C02027.

[13] A. Affolder et al., DC-DC converters with reduced mass for trackers at the HL-LHC, JINST 6 (2011) C11035.

[14] P. Moreira et al., The GBT Project, CERN-2009-006, https://cds.cern.ch/record/1235836. 
[15] L. A. M. Wiik-Fuchs et al., First Double-Sided End-Cap Strip Module for the ATLAS High-Luminosity Upgrade, Topical Workshop on Electronics for Particle Physics, PoS (TWEPP2018) 015.

[16] R. Diener et al., The DESY II test beam facility, Nucl. Instrum. Meth. A 922 (2019) 265.

[17] H. Jansen et al., Performance of the EUDET-type beam telescopes, EPJ Tech Instrum 3 (2016).

[18] Y. Liu et al., EUDAQ2-A flexible data acquisition software framework for common test beams, JINST 14 (2019) P10033.

[19] H. Perrey, EUDAQ and EUTelescope: Software Frameworks for Test Beam Data Acquisition and Analysis, Technology and Instrumentation in Particle Physics, PoS (TIPP2014) 353.

[20] A. J. Barr et al., Beamtests of ATLAS SCT Modules in August and October 2001, ATL-INDET-2002-024, http://cds.cern.ch/record/685412 